\title{
Research Article \\ Stability of Fluid Flow through a Channel with Flexible Walls
}

\author{
Marianna A. Shubov $1 D$ and Madeline M. Edwards \\ Department of Mathematics and Statistics, University of New Hampshire, 33 Academic Way, Durham 03825, NH, USA \\ Correspondence should be addressed to Marianna A. Shubov; marianna.shubov@gmail.com
}

Received 1 September 2020; Revised 20 February 2021; Accepted 26 February 2021; Published 13 March 2021

Academic Editor: Sergejs Solovjovs

Copyright (c) 2021 Marianna A. Shubov and Madeline M. Edwards. This is an open access article distributed under the Creative Commons Attribution License, which permits unrestricted use, distribution, and reproduction in any medium, provided the original work is properly cited.

\begin{abstract}
In the present paper, we summarize the results of the research devoted to the problem of stability of the fluid flow moving in a channel with flexible walls and interacting with the walls. The walls of the vessel are subject to traveling waves. Experimental data show that the energy of the flowing fluid can be transferred and consumed by the structure (the walls), inducing "traveling wave flutter." The problem of stability of fluid-structure interaction splits into two parts: (a) stability of fluid flow in the channel with harmonically moving walls and (b) stability of solid structure participating in the energy exchange with the flow. Stability of fluid flow, the main focus of the research, is obtained by solving the initial boundary value problem for the stream function. The main findings of the paper are the following: (i) rigorous formulation of the initial boundary problem for the stream function, $\psi(x, y, t)$, induced by the fluid-structure interaction model, which takes into account the axisymmetric pattern of the flow and "no-slip" condition near the channel walls; (ii) application of a double integral transformation (the Fourier transformation and Laplace transformation) to both the equation and boundary and initial conditions, which reduces the original partial differential equation to a parameter-dependent ordinary differential equation; (iii) derivation of the explicit formula for the Fourier transform of the stream function, $\widetilde{\psi}(k, y, t)$; (iv) evaluation of the inverse Fourier transform of $\widetilde{\psi}(k, y, t)$ and proving that reconstruction of $\psi(x, y, t)$ can be obtained through a limiting process in the complex $k$-plane, which allows us to use the Residue theorem and represent the solution in the form of an infinite series of residues. The result of this research is an analytical solution describing blood flowing through a channel with flexible walls that are being perturbed in the form of a traveling wave.
\end{abstract}

\section{Introduction}

In the present paper, we formulate a number of results obtained for a mathematical model describing incompressible fluid flowing through a relatively long channel with flexible walls. The model has originated in a biological setting of blood moving through large arteries (or veins), whose walls are subject to traveling waves. Experiments show that, under some conditions, the energy of the fluid flow can be transferred and then consumed by the structure (the walls), which initiate "traveling wave flutter" [1].

We focus on the problem of stability, which splits into two parts: (i) stability of the fluid flow in the channel, whose walls undergo axisymmetric harmonic movement, and (ii) the stability of the channel wall structure, which is participating in the energy exchange with the flow. The present paper deals with the first part of the problem; that is, we provide a closed-form solution of the problem of stability of the fluid flow in the channel with harmonically moving walls. Our forthcoming work will be devoted to the structure response on the pressure changes from the flow. The main achievement of the present paper is the derivation of the set of the explicit formulas for the flow velocities, which allows us to answer the question on the flow stability. We expect that the results obtained for two-dimensional configuration will be relevant to the investigation of flutter-type instabilities of collapsible tubes in three-dimensional setting.

Before we present our results and discuss their novelty, we briefly discuss recent research works containing either new important results or new analytic techniques. The authors of [2-4], Case and Shivamoggi, consider the stability problem for inviscid incompressible fluid flowing between infinite parallel plates. Carpenter and Garrad [5] investigate flow-induced surface instabilities using a thin elastic plate 
model supported on a spring foundation. Kumaran [6, 7] investigates the stability problem for the fluid flowing through flexible tubes such as blood vessels. The results derived in [6] on hydrodynamic stability are similar to the classical results of Drazin and Reid [8]. Heil and Jensen [9] carry out analysis of steady flows in three-dimensional collapsible tubes and obtain the results that cannot be predicted by analyzing the lower-dimensional models.

In a series of works, different authors investigate nonNewtonian flows in different biological and medical settings. The authors of [10], Abdelsalam and Bhatti, derive a model of bioconvection flow and heat transfer through the space between two infinite coaxial conduits. They use a mathematical model and show that pressure fluctuations and outer and inner tube friction could lead to the blood clots and endoscopic effects (see also work [11] by Elmaboud and Abdelsalam). In paper [12], Abdelsalam and Sohail investigate the heat and mass transfer in 3-dimensional setting of viscous fluid flow containing microorganisms over a stretched membrane. The authors of [13], Abdelsalam et al., focus on the model of blood stream in the presence of electroosmotic forces in the arteries having both aneurysm and stenosis. The model is supposed to be useful in medical pumps for drug delivery systems incorporating nanoparticles. In [14], Abumandour et al. analyze the effects of viscosity and magnetohydrodynamics of the peristalsis of nanofluid using both analytical and numerical mathematical tools. The authors of [15], Bhatti et al., provide an analytical study of a model of swimming microorganisms in a nonNewtonian blood-based nanofluid moving along an anisotropically narrowing artery. They take into account the rheology as a non-Newtonian fluid. In the editorial paper [16], Bhatti et al. outline state-of-the-art research of computational fluid dynamics related to biologically inspired models. The authors of [17], Eldesoky et al., and Eldesoky et al. of [18] study the effect of wall slip condition and heat transfer on peristaltic inflow of magnetohydrodynamics Newtonian fluid with suspended particles in a catheterized tube. Sadat and Abdelsalam [19] analyze physical characteristics of incompressible hybrid nanofluid (composed of two different types of nanoparticles) in an annulus with flexible boundaries. The authors present a comparison of the results obtained for "standard" nanofluids and hybrid nanofluids. The authors suggest that such a model can be instrumental for the study of the metabolic structures playing an important role in heat sources inside the human body. The authors of [20], Sohail et al., investigate the properties of the boundary layer flow of Casson liquid over a linearly elongated surface in porous medium. A coupled system of nonlinear ordinary differential equations describing the model is solved numerically and interpreted graphically.

The authors of [21], Aittokallio et al., propose a mathematical model describing the operation of the lungs (both the inspiratory and the expiratory phases). Beck et al. [22] discuss the acoustic properties of snoring sounds and identify two different patterns: the "simple-waveform" snores and the "complex-waveform" snores. Grotberg and Gavriely [23] focus on flow-induced flutter oscillations producing wheezing sounds. Their model predicts the critical fluid speed that initiates flutter of the wall. Self-excited oscillations of collapsible lung airways are responsible for respiratory wheezes during forced expiration, for speech production during flow-induced vibrations of the vocal chords, and for snoring sounds during deformation of the soft palate and pharyngeal wall [9, 21-28]. Thin-walled circular shell structures containing or immersed in flowing fluid may be found in many engineering and biomechanical systems [29-32]. In many applications, the length of the flexible tube is shorter than the entrance length of the flow. Larose and Grotberg [33] study the fluid-elastic (flutter) instability with a developing flow in a compliant channel. Païdoussis and Li [34] present a comprehensive study of different types of pipes conveying fluid (straight and curved pipes, cantilevered pipes, and supported pipes). The authors of [35, 36], Païdoussis, Semler, and Wadham-Gagnon, present an extended review on the stability of aspiring pipes. Païdoussis and Denise [31] provide an analytical model and experimental results for clamped-clamped and clamped-free shells conveying inviscid fluid. Amabili et al. [37] discuss the nonlinear dynamics and stability of circular cylindrical shells containing fluid flow and show that the system loses stability by divergence.

Huang [1] studies the collapse and subsequent self-excited oscillation of compliant tubes conveying fluids. The author considers a two-dimensional, inviscid, shear flow in a flexible channel of infinite length subject to linear traveling varicose waves. Huang observes (i) the reversal of the collapsing tendency of compliant fluid passages and (ii) the fact that the fluid pressure always has a component in phase with the wave slope causing wave drag and energy transfer from the flow to the waves (a mechanism for traveling wave flutter). In [38], Huang carries out analysis of a coupled system describing the Poiseuille flow interacting with a tensioned membrane of a finite length. The author shows that (i) flutter and divergence may occur at similar flow velocities and may coexist. (ii) The elastic waves over a membrane, having a standing wave pattern, can be decomposed into upstream traveling waves and downstream traveling waves. The downstream component is responsible for energy transfer from the fluid to the wall.

Now we are in a position to outline briefly the content of the present paper. Section 2 is devoted to the formulation of the initial boundary value problem describing the dynamics of pressure perturbation in the horizontal channel. The upper boundary condition reflects an assumption on the axial symmetry of the unperturbed problem with respect to the centerline of the channel (see equation (6)). We consider quite general behavior of the lower boundary of the channel and derive the equation that relates the vertical component of the flow velocity and the velocity of the points of a solid boundary of the channel (see equation (8)). We emphasize that we do not impose any restrictions on the function describing the elevation of the lower boundary, which means that equation (8) can be used to analyze nonharmonic movement of the channel boundary. In the present work, we assume that the channel is a horizontal tube with the following properties of the walls reflecting the physical origin of 
the model. There exists a large positive number $R(R \gg 1)$ such that within the symmetric interval $(-R, R)$ the wall is flexible and the lower boundary moves "almost harmonically" in accordance with the law:

$$
g(x, t)=C_{0} e^{i \omega(x-c t)}, \quad \omega>0, x \in[-R, R],
$$

where $\omega$ is the wavenumber and $c$ is the speed of the wave crest propagation; $g(x, t)$ denotes the transverse displacement of the channel wall at location $x$ and moment in time $t$; $C_{0}$ is a small positive constant. We also assume that (1) there exists a small positive number, $r: r \ll R$, and $r$ is such that, outside the interval $[-R-r, R+r]$, the wall is rigid; that is, the vertical displacement on $\mathbb{R} \backslash[-R-r, R+r]$ is zero; and (2) there exists a smooth function, $g_{0}(x)$, which governs the transition of displacement $g(x, t)$ from formula (1) to zero; that is, we set

$$
\begin{aligned}
g(x, t)= & g_{0}(x) e^{i \omega(x-c t)}, \quad\left|g_{0}(x)\right| \ll 1, \omega>0,-R-r \leq x \\
& \leq R+r, r \ll 1,
\end{aligned}
$$

with $\operatorname{supp}\left\{g_{0}(x)\right\} \in[-R-r, R+r]$ and $g_{0}(x)=g_{0}(0)=C_{0}$ for $x \in[-R, R]$ (see Figure 1 ).

Finally, we present the initial boundary value problem (IBVP) given by (10)-(13) for the stream function $\psi(x, y, t)$, in which the harmonic behavior of the wall is addressed. We recall that if one knows the stream function, then the components of the velocity vector can be reconstructed [8] (see formulae (3)).

In Section 3, we provide a modification of the IBVP given by (10)-(13) and reduce it to a more tractable form. First, we apply the Fourier transformation to the equation and the boundary and initial conditions. As a result, we obtain an equation in which the partial derivatives, with respect to the $x$-variable, are replaced with polynomials with respect to the Fourier transform parameter, $k$ (see equation (19)). Second, we apply to equation (19) the Laplace transformation with respect to the time variable, $t$. The second integral transformation allows us to take into account the explicit expression for the unperturbed flow velocity profile, $U(y)$. After incorporating $U(y)$, we apply the inverse Laplace transformation and reduce IBVP (10)-(13) to the new formulation (24)-(27). The main equation (24) is an ordinary differential equation with respect to the variable $y$ (the width of the channel) with the complex parameter $k$ (the result of the Fourier transformation) entering both the equation and the boundary conditions. This equation has time-dependent right-hand side and nonhomogeneous boundary conditions. We conclude this section with introducing and explicitly solving the boundary problem for the corresponding stationary solution (31). Using the stationary solution, we reduce IBVP (24)-(27) to a new one having homogeneous boundary conditions (34)-(36). The rest of Section 3 is devoted to the construction of the inverse operator to the second-order differential operator with the Dirichlet boundary conditions. The inverse operator is an integral operator, whose kernel is Green's function. We derive an explicit formula for Green's function and present a

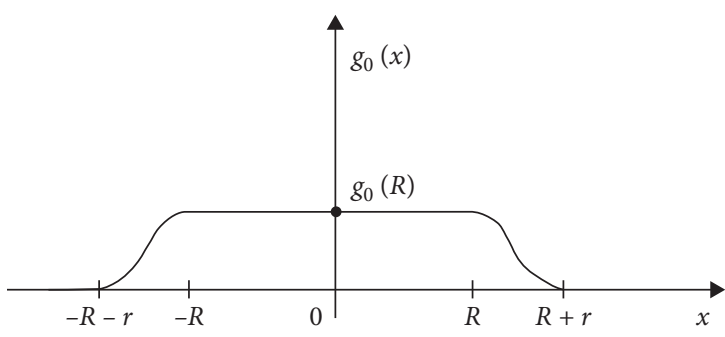

Figure 1: The amplitude profile function.

closed-form expression for the Fourier transform of the stream function denoted by $\widetilde{\psi}(k, y, t)$ (see formula (41)).

To examine the behavior of the stream function as a function of variable $x$, we have to evaluate the inverse Fourier transform of (41). Section 4 contains the main technical results of the paper.

The technical tools used in proving the main results are as follows. The Fourier transform of the stream function, $\widetilde{\psi}(k, y, t)$, is represented as a sum of three terms (see formula (41)). The inverse Fourier transform, which is the stream function $\psi$ itself, also contains three terms denoted by $I_{1}, I_{2}$, and $I_{3}$ (see formulae (43)-(45)). The main results of the paper are demonstrated in Theorems 1-3. These theorems provide the desired representation for the stream function as a function of time and space. To obtain such representations, one has to split $\widetilde{\psi}(k, y, t)$ into several terms and prove that each term, containing the improper integral with respect to $k$, can be extended into the complex $k$-plane and evaluated by using the Residue theorem (the detailed derivation is presented in our paper [39]). The series (46), (62), and (63) converge at exponential rates. It means that one can take only several terms from each series and attain the desired level of accuracy, which is important for practical applications of the obtained formulae.

\section{Statement of the Problem}

We consider a two-dimensional, inviscid, incompressible shear flow through a long channel with elastic walls. The two-channel walls undergo varicose heaving motions of small constant amplitudes in the form of a wave traveling in the direction of the flow, that is, along the $x$-axis (see Figure 2). Using dimensionless variables, we set the channel height equal to two units and assume that the undisturbed flow is the symmetrization of the Couette flow with respect to the axis $y=1$. Channel wall movements induce pressure disturbances within the fluid flow. Our goal is to provide the analytical representation of the pressure distribution.

Based on the axial symmetry of the model, the 3-dimensional problem can be reduced to the 2-dimensional problem. We introduce the following 2-dimensional vector field: let $\mathbf{q}(x, y, t)=(U(y)+u(x, y, t), v(x, y, t))^{T}$ be the velocity vector with $U(y)$ (undisturbed flow profile) being the given function and $u(x, y, t)$ and $v(x, y, t)$ being the perturbations.

The incompressibility condition, $(\nabla \cdot \mathbf{q})(x, y, t)=0$ written in terms of $u(x, y, t)$ and $v(x, y, t)$, has the form $u_{x}(x, y, t)+v_{y}(x, y, t)=0$. As is well known, this equation 


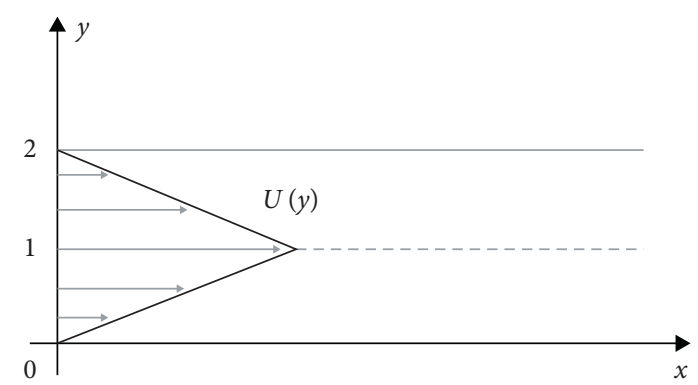

FIgURE 2: Symmetric undisturbed flow profile, $U(y)$.

means that there exists a scalar function $\psi(x, y, t)$ (called the stream function) defined on the domain $(x, y) \in \Omega \in \mathbb{R} \times$ $[0,1]$ such that

$$
\begin{aligned}
& u(x, y, t)=\psi_{y}(x, y, t), \\
& v(x, y, t)=-\psi_{x}(x, y, t)
\end{aligned}
$$

The Euler momentum equation written for $\mathbf{q}(x, y, t)$ generates a nonlinear system of two equations with respect to $u(x, y, t)$ and $v(x, y, t)$. We eliminate all nonlinear terms in the aforementioned system and rewrite them in terms of the stream function. Taking into account the symmetric modification of the Couette flow, that is, $U(y)=y, 0 \leq y \leq 1$, we obtain a system of two equations:

$$
\begin{array}{r}
\psi_{y t}(x, y, t)+y \psi_{x y}(x, y, t)-\psi_{x}(x, y, t)+p_{x}(x, y, t)=0 \\
\psi_{x t}(x, y, t)+y \psi_{x x}(x, y, t)-p_{y}(x, y, t)=0 .
\end{array}
$$

Eliminating pressure terms $\left(p_{x}\right.$ and $\left.p_{y}\right)$, we obtain the following partial differential equation for the stream function:

$$
\left(\frac{\partial}{\partial t}+y \frac{\partial}{\partial x}\right)\left(\psi_{x x}(x, y, t)+\psi_{y y}(x, y, t)\right)=0 .
$$

2.1. The Boundary Conditions. Due to the axial symmetry of the model, there is no vertical flow across the centerline $y=1$; that is,

$$
v(x, 1, t)=-\psi_{x}(x, 1, t)=0 .
$$

We consider quite general behavior of the lower boundary of the channel and derive the equation that relates the vertical component of the flow velocities and the velocities of the points of a solid boundary of the channel. Let the vertical displacement of the lower wall at a position, $x$, and at a moment in time, $t$, be given by the equation $y=g(x, t)$. It is proven in paper [39] that, to satisfy the requirement that the normal components of the fluid velocity and the wall velocity are equal, the following boundary condition at $y=0$ must be satisfied:

$$
g_{t}(x, t)=-[U(y)+u(x, y, t)] g_{x}(x, t)+v(x, y, t) .
$$

Equation (7) can be used to analyze nonharmonic movement of the channel boundary. We assume that $g(x, t)$ is a function of a small amplitude and consider linear asymptotic approximations: $U(y)=U(0)+y U_{y}(0)+\mathcal{O}\left(y^{2}\right)$, $u(x, y, t)=u(x, 0, t)+y u_{y}(x, 0, t)+\mathcal{O}\left(y^{2}\right)$, and $v(x, y, t)=$ $v(x, 0, t)+y v_{y}(x, 0, t)+\mathscr{O}\left(y^{2}\right)$. Keeping only the zero-order terms, we obtain the following approximation for the boundary condition of (7):

$$
g_{t}(x, t)+[U(0)+u(x, 0, t)] g_{x}(x, t)=v(x, 0, t) .
$$

Taking into account equation (2) for $g(x, t)$ and the fact that in our model $u_{x}(x, 0, t)=0$ and $U(0)=0$, we rewrite (8) in terms of the stream function as

$$
\psi_{x}(x, 0, t)=i \omega c g(x, t) .
$$

Note that, in the problem with a rigid wall channel, we would have had the condition $\psi_{x}(x, 0, t)=0$. Thus, in the present paper, we focus on the following initial boundary value problem (IBVP) for the stream function:

$$
\begin{gathered}
\left(\frac{\partial}{\partial t}+y \frac{\partial}{\partial x}\right)\left(\psi_{x x}(x, y, t)+\psi_{y y}(x, y, t)\right)=0 \\
\psi_{x}(x, 0, t)=i \omega c g(x, t) \\
\psi_{x}(x, 1, t)=0 \\
\psi(x, y, 0)=F(x, y) .
\end{gathered}
$$

\section{Reformulation of IBVP Using Fourier and Laplace Integral Transformations}

We apply two integral transformations to the IBVP (10)-(13) in order to reduce the IBVP involving a partial differential equation to the boundary value problem involving a parameter-dependent ordinary differential equation. If $\widetilde{\psi}(k, y, t)$ denotes the Fourier transform of the stream function, then applying the Fourier transformation to equation (10) yields

$$
-k^{2} \widetilde{\psi}_{t}(k, y, t)+\widetilde{\psi}_{y y t}(k, y, t)-i k^{3} y \widetilde{\psi}(k, y, t)+i k y \widetilde{\psi}_{y y}(k, y, t)=0
$$

Let $\Psi(k, y, \lambda)$ be the Laplace transform of $\widetilde{\psi}(k, y, t)$ with respect to time variable, $t$. Applying the Laplace transformation to both sides of equation (14) yields

$$
\left(\frac{\partial^{2}}{\partial y^{2}}-k^{2}\right) \Psi(k, y, \lambda)=\frac{1}{\lambda+i k y}\left(\frac{\partial^{2}}{\partial y^{2}}-k^{2}\right) \widetilde{\psi}(k, y, 0),
$$

where $\widetilde{\psi}(k, y, 0)$ is the Fourier transform of the initial state. Notice that the only influence of the Couette-like flow is the " $y$ " term in the denominator on the right-hand side of (15). If instead of Couette-like profile we have an arbitrary axisymmetric profile, $U(y)$, then, in place of " $y$," we would 
have had the function, $U(y)$, and thus have a factor of $(\lambda+i k U(y))^{-1}$ in equation (15). The unknown function, $\Psi(k, y, \lambda)$ in (15), being a function of $y, 0 \leq y \leq 1$, depends on two complex parameters, $k$ and $\lambda$.

Applying the inverse Laplace transformation to both sides of equation (15) yields

$$
\begin{gathered}
\frac{1}{2 \pi i} \int_{\gamma}\left(\frac{\partial^{2}}{\partial y^{2}}-k^{2}\right) \Psi(k, y, \lambda) e^{\lambda t} \mathrm{~d} \lambda=\frac{1}{2 \pi i} \\
\int_{\gamma} \frac{e^{\lambda t}}{\lambda+i k y}\left(\frac{\partial^{2}}{\partial y^{2}}-k^{2}\right) \tilde{\psi}(k, y, 0) \mathrm{d} \lambda,
\end{gathered}
$$

where the contour of integration can be taken any vertical line, located in the open right half-plane of the complex $\lambda$-plane (see Figure 3).

Closing the contour of integration in the $\lambda$-complex plane and using the Residue theorem to evaluate the contour integral at the right-hand side of equation (16), we obtain the desired form of the differential equation:

$$
\left(\frac{\partial^{2}}{\partial y^{2}}-k^{2}\right) \widetilde{\psi}(k, y, t)=e^{-i k y t}\left(\frac{\partial^{2}}{\partial y^{2}}-k^{2}\right) \widetilde{\psi}(k, y, 0) .
$$

However, we do not have the boundary conditions for $\widetilde{\psi}(k, y, t)$; we have the boundary conditions only for $\psi_{x}(k, y, t)$ when $y=0$ and $y=1$ (see (11) and (12)). Since $\psi_{x}(x, 1, t)=0$, we immediately obtain the boundary condition at $y=1, \widetilde{\psi}(k, 1, t)=0$. To derive the boundary condition at $y=0$, we have to specify the properties of the force profile function, $g_{0}(x)$ (see (9)). According to the physical origin of the model, the channel walls are flexible for $|x| \leq R+r$ and rigid for $|x|>R+r$.

Assumption 1. The amplitude function, $g_{0}(x)$, is a continuously differentiable, even function with a compact support, such that (see Figure 1)

$$
\begin{aligned}
& g_{0}(x)=0, \quad \text { for } x \in \mathbb{R} /[-R-r, R+r], R \gg 1, r \ll 1, \\
& g_{0}(x)=g_{0}(R) \ll 1, \quad \text { for } x \in[-R, R] .
\end{aligned}
$$

Collecting equation (17), boundary condition at $y=1$, and evaluating the Fourier transformation of the boundary condition (11), we obtain the following reformulation of the IBVP (10)-(13) for $\widetilde{\psi}(k, y, t)$ :

$$
\begin{aligned}
& \left(\frac{\partial^{2}}{\partial y^{2}}-k^{2}\right) \widetilde{\psi}(k, y, t)=e^{-i k y t}\left(\frac{\partial^{2}}{\partial y^{2}}-k^{2}\right) \widetilde{\psi}(k, y, 0), \\
& \widetilde{\psi}(k, 0, t)=-\frac{2 \omega c}{k} e^{-i \omega c t} \int_{R}^{R+r} g_{0}^{\prime}(\xi) \frac{\sin (\xi(k-\omega))}{k-\omega} \mathrm{d} \xi, \\
& \widetilde{\psi}(k, 1, t)=0, \\
& \widetilde{\psi}(k, y, 0)=\widetilde{F}(k, y) .
\end{aligned}
$$

IBVP (19)-(22) is the main object of interest in the rest of the paper.

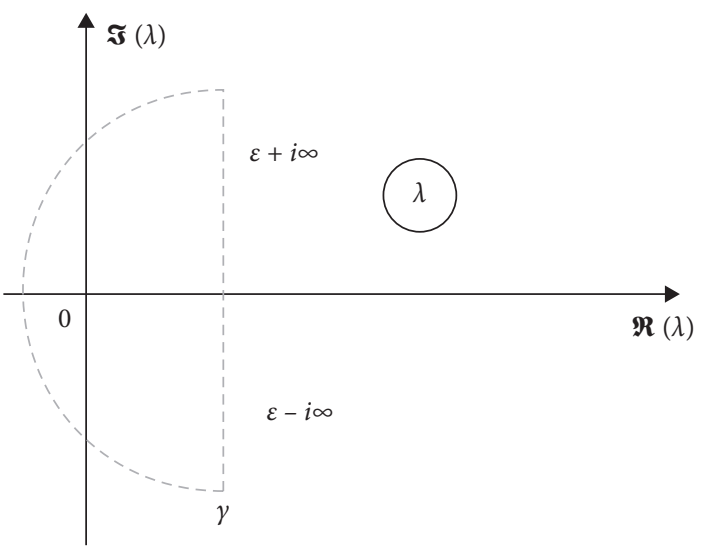

Figure 3: Contour for inversion of Laplace transform.

3.1. Reduction of Problems (19)-(22) to the Problem with TimeIndependent Boundary Conditions. Let us introduce a new function:

$$
Y(k, y, t)=e^{i \omega c t} \widetilde{\psi}(k, y, t) .
$$

Since $Y(k, y, 0)=\widetilde{\psi}(k, y, 0)$, the problem for $Y(k, y, t)$ can be represented in the form

$$
\left(\frac{\partial^{2}}{\partial y^{2}}-k^{2}\right) Y(k, y, t)=e^{-i(k y-\omega c) t}\left(\frac{\partial^{2}}{\partial y^{2}}-k^{2}\right) Y(k, y, 0)
$$

$$
\begin{aligned}
Y(k, 0, t) & =-\frac{2 \omega c}{k} \int_{R}^{R+r} g_{0}^{\prime}(\xi) \frac{\sin (\xi(k-\omega))}{k-\omega} \mathrm{d} \xi \\
Y(k, 1, t) & =0 \\
Y(k, y, 0) & =\widetilde{F}(k, y) .
\end{aligned}
$$

To reduce the IBVP (24)-(27) to the IBVP with homogeneous boundary conditions, we introduce the steadystate solution, $Y_{0}(k, y)$, which satisfies the following boundary value problem:

$$
\begin{gathered}
\left(\frac{\partial^{2}}{\partial^{2} y}-k^{2}\right) Y_{0}(k, y)=0, \\
Y_{0}(k, 1)=0, \\
Y_{0}(k, 0)=-\frac{2 \omega c}{k} \int_{R}^{R+r} g_{0}^{\prime}(\xi) \frac{\sin (\xi(k-\omega))}{k-\omega} \mathrm{d} \xi .
\end{gathered}
$$

This problem has a unique solution that can be given by an explicit formula:

$$
Y_{0}(k, y)=-\frac{2 \omega c \sinh (k(1-y))}{k \sinh (k)} \int_{R}^{R+r} g_{0}^{\prime}(\xi) \frac{\sin (\xi(k-\omega))}{k-\omega} \mathrm{d} \xi .
$$

It can be readily seen that the steady-state solution satisfies the initial boundary value problem (24)-(27), in which the initial state is simply $Y_{0}(k, y)$. 
Assumption 2. We assume that the initial state of the system is a function whose Fourier transform is a small perturbation of the steady-state solution; that is,

$$
\widetilde{F}(k, y)=\tilde{f}(k, y)+Y_{0}(k, y),
$$

where $\tilde{f}(k, y)$ is a smooth compactly supported function of $y$. Now, let us introduce a new function, $Z(k, y, t)$, which satisfies the zero boundary conditions; that is,

$$
Z(k, y, t)=Y(k, y, t)-Y_{0}(k, y) .
$$

It can be verified by straightforward calculations that the IBVP for $Z(k, y, t)$ can be written in the following form:

$$
\begin{aligned}
\left(\frac{\partial^{2}}{\partial y^{2}}-k^{2}\right) Z(k, y, t) & =e^{-i(k y-\omega c) t}\left(\frac{\partial^{2}}{\partial y^{2}}-k^{2}\right) \widetilde{\psi}(k, y, 0) \\
Z(k, 0, t) & =Z(k, 1, t)=0 \\
Z(k, y, 0) & =\tilde{f}(k, y)
\end{aligned}
$$

3.2. Solving Problems (34)-(36) via Green's Function. We construct an explicit solution of equation (34) satisfying the Dirichlet boundary conditions (35). The initial condition (36) is incorporated into equation (34) in which $\widetilde{\psi}(k, y, 0)=\widetilde{f}(k, y)$.
As is known [40,41], the solution of the boundary problem,

$$
W^{\prime \prime}(k, y)-k^{2} W(k, y)=\Phi(k, y), \quad W(k, 0)=W(k, 1)=0,
$$

can be given in terms of Green's function as

$$
W(k, y)=\int_{0}^{1} G(\eta, y) \Phi(\eta, k) \mathrm{d} \eta .
$$

The following properties must be satisfied for $G(\eta, y)[40,41]$ : (i) symmetry, that is, $G(\eta, y)=G(y, \eta)$; (ii) continuity, that is, $G(\eta, y) \longrightarrow G(y, y)$ as $\eta \longrightarrow y$; (iii) a unit jump derivative, that is, $\left.G_{y}(\eta, y)\right|_{y=\eta+0}$ $\left.G_{y}(\eta, y)\right|_{y=\eta-0}=1$; (iv) the boundary conditions: $G(\eta, 0)=G(\eta, 1)=0$; (v) the equation: $G_{y y}(\eta, y)-k^{2} G$ $(\eta, y)=\delta(\eta-y)$ with $\delta(\cdot)$ being the Dirac delta function.

It can be verified directly that the function given by explicit formula,

$$
G(\eta, y)=\frac{1}{k \sinh (k)} \begin{cases}-\sinh (k(1-\eta)) \sinh (k y), & y<\eta, \\ -\sinh (k \eta) \sinh (k(1-y)), & y>\eta,\end{cases}
$$

satisfies all properties (i)-(v). Using this Green's function, we obtain the following solution of systems (34) and (35):

$$
\begin{aligned}
Z(k, y, t)= & e^{i \omega c t} \int_{0}^{1} e^{-i k \eta t} G(y, \eta)\left(\frac{\partial^{2}}{\partial \eta^{2}}-k^{2}\right) \widetilde{\psi}(k, \eta, 0) \mathrm{d} \eta \\
= & -e^{i \omega c t} \frac{\sinh (k(1-y))}{k \sinh (k)} \int_{0}^{y} e^{-i k \eta t} \sinh (k \eta)\left(\frac{\partial^{2}}{\partial \eta^{2}}-k^{2}\right) \widetilde{\psi}(k, \eta, 0) \mathrm{d} \eta \\
& -e^{i \omega c t} \frac{\sinh (k y)}{k \sinh (k)} \int_{y}^{1} e^{-i k \eta t} \sinh (k(1-\eta))\left(\frac{\partial^{2}}{\partial \eta^{2}}-k^{2}\right) \widetilde{\psi}(k, \eta, 0) \mathrm{d} \eta,
\end{aligned}
$$

which yields the desired result for $\widetilde{\psi}(k, y, t)=e^{-i \omega c t}\left[Z(k, y, t)+Y_{0}(k, y)\right]$ :

$$
\begin{aligned}
\widetilde{\psi}(k, y, t)= & -\frac{\sinh (k(1-y))}{k \sinh (k)} \int_{0}^{y} e^{-i k \eta t} \sinh (k \eta)\left(\frac{\partial^{2}}{\partial \eta^{2}}-k^{2}\right) \widetilde{\psi}(k, \eta, 0) \mathrm{d} \eta \\
& -\frac{\sinh (k y)}{k \sinh (k)} \int_{y}^{1} e^{-i k \eta t} \sinh (k(1-\eta))\left(\frac{\partial^{2}}{\partial \eta^{2}}-k^{2}\right) \widetilde{\psi}(k, \eta, 0) \mathrm{d} \eta \\
& -e^{-i \omega c t} \frac{2 \omega c \sinh (k(1-y))}{k \sinh (k)} \int_{R}^{R+r} g_{0}^{\prime}(\xi) \frac{\sin (\xi(k-\omega))}{k-\omega} \mathrm{d} \xi .
\end{aligned}
$$




\section{Evaluation of the Inverse Fourier Transforms}

We need to provide the space-time representation for the vertical component of the velocity perturbation $v(x, y, t)$, which is related to the derivative of the stream function by formula (6). It means that, in fact, we are looking for the inverse Fourier transform of the function $k \widetilde{\psi}(k, y, t)$. It is convenient to introduce the following notation for the inverse Fourier transform of the stream function:

$$
i \psi_{x}(x, y, t)=\frac{1}{2 \pi} \int_{-\infty}^{\infty} e^{i k x} k \widetilde{\psi}(k, y, t) \mathrm{d} k \equiv I_{1}+I_{2}+I_{3},
$$

where

$$
\begin{aligned}
& I_{1}=-\frac{c \omega}{\pi} e^{-i \omega c t} \int_{-\infty}^{\infty} e^{i k x}\left[\frac{\sinh (k(1-y))}{\sinh (k)} \int_{R}^{R+r} g_{0}^{\prime}(\xi) \frac{\sin (\xi(k-\omega))}{k-\omega} \mathrm{d} \xi\right] \mathrm{d} k, \\
& I_{2}=-\frac{1}{2 \pi} \int_{-\infty}^{\infty} e^{i k x}\left[\frac{\sinh (k(1-y))}{\sinh (k)} \int_{0}^{y} e^{-i k \eta t} \sinh (k \eta)\left(\frac{\partial^{2}}{\partial \eta^{2}}-k^{2}\right) \tilde{f}(k, \eta) \mathrm{d} \eta\right] \mathrm{d} k, \\
& I_{3}=-\frac{1}{2 \pi} \int_{-\infty}^{\infty} e^{i k x}\left[\frac{\sinh (k y)}{\sinh (k)} \int_{y}^{1} e^{-i k \eta t} \sinh (k(1-\eta))\left(\frac{\partial^{2}}{\partial \eta^{2}}-k^{2}\right) \tilde{f}(k, \eta) \mathrm{d} \eta\right] \mathrm{d} k .
\end{aligned}
$$

Our first result is concerned with the integral $I_{1}$. This integral is, in fact, the solution of the IBVP with $f(x, y)=0$.
Theorem 1. The following explicit formula holds for the term $I_{1}$ from the decomposition (42):

$$
\begin{aligned}
I_{1}= & -\omega c e^{i \omega(x-c t)} g_{0}(R) \frac{\sinh (\omega(1-y))}{\sinh (\omega)}-2 \omega c e^{-i \omega c t} \sum_{m=1}^{\infty} \frac{\sin \pi m y}{\pi^{2} m^{2}+\omega^{2}} \\
& \times \int_{R}^{R+r} \mathrm{~d} \xi g_{0}^{\prime}(\xi) e^{-\pi m \xi}[\pi m \cosh (\pi m x+i \xi \omega)-i \omega \sinh (\pi m x+i \xi \omega)],
\end{aligned}
$$

with the series being absolutely convergent for any $x \in(-R, R), 0<y<1$, and $-\infty<t<\infty$.

4.1. Remarks on the Proof of Theorem 1. We outline the analytical tools needed to investigate the structure of $I_{1}$. Using Fourier and Laplace transforms, we obtain the following formula for $I_{1}$ (see (43)):

$$
I_{1}=-\frac{c \omega}{\pi} e^{-i \omega c t} \int_{R}^{R+r} \mathrm{~d} \xi g_{0}^{\prime}(\xi) \int_{-\infty}^{\infty} \mathrm{d} k e^{i k x} \frac{\sinh (k(1-y))}{\sinh (k)} \frac{\sin (\xi(k-\omega))}{k-\omega},
$$

where $R$ and $r$ are introduced in (2) in the description of amplitude function. Formula (47) is not convenient for the analysis of the qualitative behavior of $I_{1}$ as a function of $x$ and $y$. For this reason, we investigate the improper integral from (47) further and evaluate it using techniques of complex analysis. To carry out the integration in (47) with respect to $k$, we split $\sin (\xi(k-\omega))$ into two exponential functions and prove that each of the two resulting integrals, which we denote by $\mathscr{I}$ and $\tilde{\mathscr{I}}$, respectively, converges in the sense of the principal value. Each integral of $\mathscr{I}$ and $\widetilde{\mathscr{I}}$ can be approximated by sequences of integrals $\mathscr{I}_{n}$ and $\tilde{\mathscr{I}}_{n}, n \longrightarrow \infty$; that is, $I_{1}=\lim _{n \rightarrow \infty}\left(\mathscr{I}_{n}+\widetilde{\mathscr{I}}_{n}\right)$. If we fix $n$, then the domain of integration for $\mathscr{I}_{n}$ (as well as $\left.\widetilde{\mathscr{I}}_{n}\right)$ is given by $\left(-\infty, \omega-\varepsilon_{n}\right) \cup\left(\omega+\varepsilon_{n}, \infty\right)$, with $\left\{\varepsilon_{n}\right\}_{n=1}^{\infty}$ being a sequence of positive numbers converging to zero. For each $n$, the domain consists of two disjoint subdomains. In turn, $\mathscr{J}_{n}$ can be represented as a limit of a sequence of integrals $\mathscr{F}_{n}^{S}$, where each $\mathscr{F}_{n}^{S}$ is defined on the domain $\left(-S, \omega-\varepsilon_{n}\right) \cup\left(\omega+\varepsilon_{n}, S\right)$; that is, $\mathscr{I}_{n}=\lim _{S \longrightarrow \infty}$ $\mathcal{F}_{n}^{S}$. For a given pair $(n, S)$, we consider a closed contour on the complex $k$-plane, obtained by connecting two segments $\left(-S, \omega-\varepsilon_{n}\right)$ and $\left(\omega+\varepsilon_{n}, S\right)$ by two semicircles (see Figure 4$), C_{S}(0)$ and $C_{\varepsilon_{n}}(\omega)$, where $C_{S}(0)$ is a semicircle centered at the origin of radius $S$ and $C_{\varepsilon_{n}}(\omega)$ is a semicircle centered at $x=\omega$ of radius $\varepsilon_{n}$.

We show that the integral along $C_{S}(0)$ tends to zero as $S \longrightarrow \infty$ and the integral along $C_{\varepsilon_{u}}(\omega)$ tends to zero as $\varepsilon_{n} \longrightarrow 0$. Thus, we show that the integral $\mathscr{I}_{n}$ can be obtained as a limit of a sequence of the closed contour integrals. Similar steps can be carried out for evaluation of the integral $\widetilde{\mathscr{I}}_{n}$ by closing the contour of its integration in the lower halfplane. In turn, closed contour integrals can be evaluated by using the Residue theorem.

As a result, we obtain that the term $I_{1}$ can be represented in the form of an infinite series given in (46). We notice that, for $|x|<R$ and $\xi>x$, the series converges at an exponential rate. Thus, to analyze the behavior of $I_{1}$ as a function of $x, y, t$, one can keep a finite number of terms in the sum and obtain the required accuracy.

To formulate the results on the integrals $I_{2}$ and $I_{3}$, we assume that $f(x, 0)=f(x, 1)=0$ and make some preliminary steps. Namely, we integrate by parts twice in formula (44) and (45) and then take the sum to have 


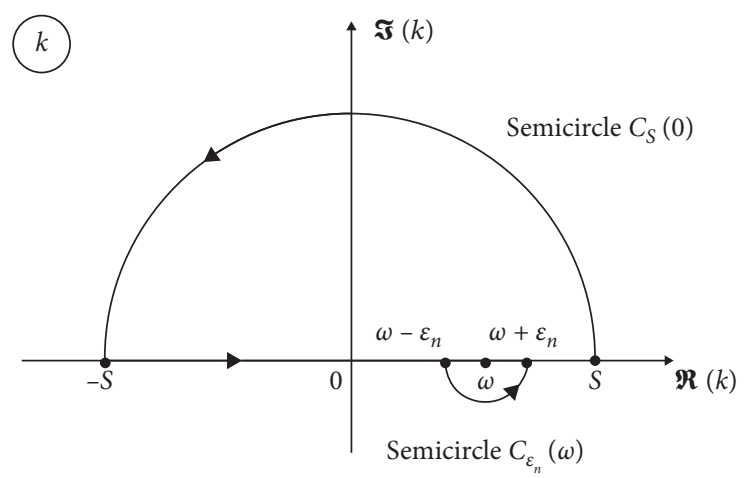

FIGURE 4: Closed contour in the upper half-plane for evaluation of $\mathscr{I}_{n}$.

$$
\begin{aligned}
-\frac{1}{2 \pi}\left(I_{2}+I_{3}\right)= & \int_{-\infty}^{\infty} \mathrm{d} k k e^{i k x} e^{-i k y t} \tilde{f}(k, y) \\
& +\int_{-\infty}^{\infty} \mathrm{d} k \frac{e^{i k x}}{\sinh (k)}\left\{\sinh (k(1-y)) \int_{0}^{y} \mathrm{~d} \eta \tilde{f}(k, \eta)\left(\frac{\partial^{2}}{\partial \eta^{2}}-k^{2}\right)\left[e^{-i k \eta t} \sinh (k \eta)\right]\right. \\
& \left.+\sinh (k y) \int_{y}^{1} \mathrm{~d} \eta \tilde{f}(k, \eta)\left(\frac{\partial^{2}}{\partial \eta^{2}}-k^{2}\right)\left[e^{-i k \eta t} \sinh (k(1-\eta))\right]\right\} .
\end{aligned}
$$

Remark 1 . One can readily check that the first integral of (48) generates the following result:

$$
-\int_{-\infty}^{\infty} \mathrm{d} k k e^{i k(x-y t)} \tilde{f}(k, y)=i f_{x}(x-y t, y) .
$$

If we fix $y$ for $0<y<1$ and consider $x$ and $t$ such that $x-y t=C$, where $f_{x}(x-y t, y)$ is some constant, then the function $f_{x}(x-y t, y)$ is also equal to a constant. It means that the perturbation velocity moves with the speed $y$ along the $x$-axis. The second improper integral in (48) can be represented as a sum $\sum_{j=1}^{4} \mathbf{I}_{j}$, where

$$
\begin{aligned}
& \mathbf{I}_{1}=\int_{0}^{y} \mathrm{~d} \eta \int_{-\infty}^{\infty} \mathrm{d} k e^{i k(x-\eta t)} \tilde{f}(k, \eta)\left(-k^{2} t^{2}\right) \frac{\sinh (k(1-y))}{\sinh (k)} \sinh (k \eta), \\
& \mathbf{I}_{2}=\int_{0}^{y} \mathrm{~d} \eta \int_{-\infty}^{\infty} \mathrm{d} k e^{i k(x-\eta t)} \tilde{f}(k, \eta)\left(-2 i k^{2} t\right) \frac{\sinh (k(1-y))}{\sinh (k)} \cosh (k \eta), \\
& \mathbf{I}_{3}=\int_{y}^{1} \mathrm{~d} \eta \int_{-\infty}^{\infty} \mathrm{d} k e^{i k(x-\eta t)} \tilde{f}(k, \eta)\left(-k^{2} t^{2}\right) \frac{\sinh (k y)}{\sinh (k)} \sinh (k(1-\eta)), \\
& \mathbf{I}_{4}=-\int_{y}^{1} \mathrm{~d} \eta \int_{-\infty}^{\infty} \mathrm{d} k e^{i k(x-\eta t)} \tilde{f}(k, \eta)\left(-2 i k^{2} t\right) \frac{\sinh (k y)}{\sinh (k)} \cosh (k(1-\eta)) .
\end{aligned}
$$

Assumption 3. Let $f(\cdot, y)$ be a smooth function with compact support; that is,

$$
\operatorname{supp}\{f(\cdot, y)\} \in\left(0, r_{0}\right), \quad r_{0}>0,
$$

such that

$$
f_{x}(x, y)=f_{x x}(x, y)=f_{x x x}(x, y)=0, \quad x=0, x=r_{0} .
$$

$$
\begin{aligned}
& \tilde{f}(k, y)=\frac{i}{k^{3}} \int_{0}^{r_{0}} \mathrm{~d} x e^{-i k x} f_{x x x}(x, y) \equiv \frac{1}{k} \\
& \text { where } g(x, y)=i f_{x x x} \\
& \text { It is convenient to present the integrals } \mathbf{I} \\
& \qquad \mathbf{I}_{2 j-1}=-t^{2} \widehat{\mathbf{I}}_{2 j-1}, j=1,2, \\
& \mathbf{I}_{2 j}=-2 i t \widehat{\mathbf{I}}_{2 j}, j=1,2 .
\end{aligned}
$$

Using Assumption 3, one can obtain that 
Using (50)-(53) and (57), we obtain the following representations for $\widehat{\mathbf{I}}_{j}, j=1,2,3,4$ :

$$
\begin{aligned}
& \widehat{\mathbf{I}}_{1}=\int_{0}^{r_{0}} \mathrm{~d} \xi \int_{0}^{y} \mathrm{~d} \eta g(\xi, \eta) \int_{-\infty}^{\infty} \mathrm{d} k e^{i k(x-\eta t-\xi)} \frac{\sinh (k(1-y)) \sinh (k \eta)}{k \sinh (k)}, \\
& \widehat{\mathbf{I}}_{2}=\int_{0}^{r_{0}} \mathrm{~d} \xi \int_{0}^{y} \mathrm{~d} \eta g(\xi, \eta) P . V . \int_{-\infty}^{\infty} \mathrm{d} k e^{i k(x-\eta t-\xi)} \frac{\sinh (k(1-y)) \cosh (k \eta)}{k \sinh (k)}, \\
& \widehat{\mathbf{I}}_{3}=\int_{0}^{r_{0}} \mathrm{~d} \xi \int_{y}^{1} \mathrm{~d} \eta g(\xi, \eta) \int_{-\infty}^{\infty} \mathrm{d} k e^{i k(x-\eta t-\xi)} \frac{\sinh (k(1-\eta)) \sinh (k y)}{k \sinh (k)}, \\
& \widehat{\mathbf{I}}_{4}=-\int_{0}^{r_{0}} \mathrm{~d} \xi \int_{y}^{1} \mathrm{~d} \eta g(\xi, \eta) P . V . \int_{-\infty}^{\infty} \mathrm{d} k e^{i k(x-\eta t-\xi)} \frac{\sinh (k y) \cosh (k(1-\eta))}{k \sinh (k)} .
\end{aligned}
$$

We evaluate these improper integrals by representing each of them as a limit of a sequence of closed contour integrals on the complex $k$-plane and then use the Residue theorem. It turns out that the derivation of the desired result for the sum $\left(\widehat{\mathbf{I}}_{1}+\widehat{\mathbf{I}}_{3}\right)$ is different from the derivation for the $\operatorname{sum}\left(\widehat{\mathbf{I}}_{2}+\widehat{\mathbf{I}}_{4}\right)$.

We have to consider two separate cases: $x \leq r_{0}$ and $x \geq r_{0}$. In what follows, we present the results for the case $x \geq r_{0}$. The case $x \leq r_{0}$ can be analyzed in a similar manner. The first result is concerned with the sum $\left(\widehat{\mathbf{I}}_{1}+\widehat{\mathbf{I}}_{3}\right)$.
Theorem 2. For $x \geq r_{0}$, the following representation is valid for $\left(\widehat{\mathbf{I}}_{1}+\widehat{\mathbf{I}}_{3}\right)$ :

$$
\widehat{\mathbf{I}}_{1}+\widehat{\mathbf{I}}_{3}=2 \pi \int_{0}^{r_{0}} \mathrm{~d} \xi \int_{0}^{1} \mathrm{~d} \eta g(\xi, \eta) \sum_{m=1}^{\infty} e^{-\pi m|x-\eta t-\xi| \sin (\pi m y) \sin (\pi m \eta)} \frac{\pi m}{\pi m} .
$$

Let $\mathfrak{G}(x, y, t)$ be defined as

$$
\mathfrak{G}(x, y, t)=-2 \pi i \int_{0}^{r_{0}} \mathrm{~d} \xi \int_{0}^{1} \mathrm{~d} \eta g(\xi, \eta) \frac{x-\eta t-\xi}{|x-\eta t-\xi|} \sum_{m=1}^{\infty} e^{-\pi m|x-\eta t-\xi|} \frac{\sin (\pi m y) \cos (\pi m \eta)}{\pi m}
$$

and then the following results are valid for the sum $\left(\widehat{\mathbf{I}}_{2}+\widehat{\mathbf{I}}_{4}\right)$.

(a) When $t \geq x / y$,

Theorem 3. For $x \geq r_{0}$, the following representations are valid for the sum $\left(\widehat{\mathbf{I}}_{2}+\widehat{\mathbf{I}}_{4}\right)$.

$$
\begin{aligned}
\widehat{\mathbf{I}}_{2}+\widehat{\mathbf{I}}_{4}= & \boldsymbol{G}(x, y, t)+2 \pi i(1-y) \int_{0}^{r_{0}} \mathrm{~d} \xi \int_{0}^{(x-\xi) / t} \mathrm{~d} \eta g(\xi, \eta) \\
& -\pi i(1-y) \int_{0}^{r_{0}} \mathrm{~d} \xi \int_{0}^{y} \mathrm{~d} \eta g(\xi, \eta)+\pi i y \int_{0}^{r_{0}} \mathrm{~d} \xi \int_{y}^{1} \mathrm{~d} \eta g(\xi, \eta) .
\end{aligned}
$$

(b) When $x / y>t \geq\left(x-r_{0}\right) / y$,

$$
\begin{aligned}
\widehat{\mathbf{I}}_{2}+\widehat{\mathbf{I}}_{4}= & \left(\mathfrak{G}(x, y, t)-2 \pi i y \int_{0}^{r_{0}} \mathrm{~d} \xi \int_{y}^{(x-\xi) / t} \mathrm{~d} \eta g(\xi, \eta)\right. \\
& +\pi i y \int_{0}^{r_{0}} \mathrm{~d} \xi \int_{y}^{1} \mathrm{~d} \eta g(\xi, \eta)+\pi i(1-y) \int_{0}^{r_{0}} \mathrm{~d} \xi \int_{0}^{y} \mathrm{~d} \eta g(\xi, \eta) .
\end{aligned}
$$

(c) When $\left(x-r_{0}\right) / y>t \geq\left(x-r_{0}\right)$, 


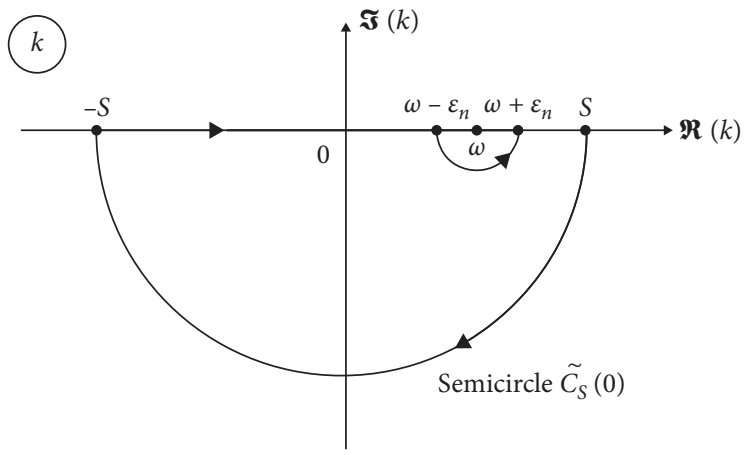

FIGURE 5: Closed contour in the lower half-plane for evaluation of $\widetilde{\mathscr{I}}_{n}$.

$$
\widehat{\mathbf{I}}_{2}+\widehat{\mathbf{I}}_{4}=\mathfrak{G}(x, y, t)-\pi i y \int_{0}^{r_{0}} \mathrm{~d} \xi \int_{y}^{1} \mathrm{~d} \eta g(\xi, \eta)+\pi i(1-y) \int_{0}^{r_{0}} \mathrm{~d} \xi \int_{0}^{y} \mathrm{~d} \eta g(\xi, \eta) .
$$

4.2. Statement on Data Availability. The present paper is concerned with an important question on stability of a fluid flow interacting with flexible channel walls. This research has a purely analytical nature; that is, it is devoted to finding a representation for a solution of the initial boundary value problem generated by Navier-Stokes equations in the case of a specifically chosen geometry of the channel and properties of the boundary. Due to the very nature of this research, we have used many mathematical tools (like techniques from the area of ordinary and partial differential equations, boundary value problems, integral transformations, improper integration, evaluation of contour integrals on the complex plane by using the Residue theorem, etc.). The obtained results are theoretical. In our forthcoming work, we are planning to compare analytical results with the experimental data. In the conclusion, we clearly indicate the major steps that have been carried out to present our research findings:

Formulation of the fluid-structure interaction model as a rigorous initial boundary value mathematical problem (IBVP) with respect to the unknown function, which is called the stream function (see partial differential equation (5)). Having the formula for the stream function, one can obtain the vertical and horizontal components of the velocity vector and then, using the Navier-Stokes equation, one can find the analytical representation of the pressure distribution.

Derivation of the boundary conditions that take into account the symmetry of the flow with respect to the centerline of the channel and the "no-slip" condition, which means that there is no relative movement between the wall and the near-wall fluid flow in the vertical direction.

Reformulation of the IBVP (10)-(13) involving partial differential equation (10) in the form of the boundary value problem (19)-(22) involving parameter-dependent ordinary differential equation (19). The reformulation is possible due to the application of double integral transformations (the Fourier and Laplace transformations) to equation (10) and corresponding boundary and initial conditions. The problem of solving an ordinary differential equation (instead of a partial differential equation) is more feasible.

Reduction of the IBVP (19)-(22) with time-dependent boundary conditions to the problem with time-independent Dirichlet boundary conditions (34)-(36) by using the steady-state solution.

Solving the problem in (34)-(36) by using the appropriate Green's function and getting an explicit expression (41) for the Fourier transform of the stream function $\widetilde{\psi}(k, y, t)$.

Reconstruction of the stream function itself, $\psi(x, y, t)$, from its Fourier transform. It is the most challenging technical step, since $\widetilde{\psi}(k, y, t)$ is represented as a sum:

$$
\widetilde{\psi}(k, y, t)=\widetilde{I}_{1}(k, y, t)+\widetilde{I}_{2}(k, y, t)+\widetilde{I}_{3}(k, y, t),
$$

and inversion of each term $I_{j}(k, y, t), j=1,2,3$, yields its own technical difficulties. To clarify all the issues occurring in the inversion of the corresponding Fourier transform, we present the detailed analysis only for the first term $\widetilde{I}_{1}$, that is, for

$$
I_{1}=-\frac{c \omega}{\pi} e^{-i \omega c t} \int_{R}^{R+r} \mathrm{~d} \xi g_{0}^{\prime}(\xi) \int_{-\infty}^{\infty} \mathrm{d} k e^{i k x} \frac{\sinh (k(1-y))}{\sinh (k)} \frac{\sin (\xi(k-\omega))}{k-\omega}
$$

Evaluation of the improper integral with respect to $k$ from the above formula for $I_{1}$ is one of the main steps in obtaining the results. In turn, this evaluation involves the following: (a) to carry out the integration 
with respect to $k$, we split the function $\sin (\xi(k-\omega))$ into two exponentials and prove that each of the two resulting integrals (denoted by $\mathscr{I}$ and $\tilde{\mathscr{I}}$, respectively) converges in the sense of principle value; (b) each integral of $\mathscr{I}$ and $\widetilde{\mathscr{I}}$ can be approximated by a sequence of closed contour integrals in the complex $k$-plane (see Figure 4 for $\mathscr{I}$ and Figure 5 for $\widetilde{\mathscr{I}}$ ); (c) each closed contour integral can be evaluated by using the Residue theorem of complex analysis and represented as a finite sum of residues; (d) passing to the limits, we arrive at formula (46) for $I_{1}$. Evaluation of the improper integrals for $\left(I_{2}+I_{3}\right)$ follows basically the same route as was used for obtaining formula (46).

The proposed problem is a biologically relevant model of blood flowing through a blood vessel. The result of this work is the analytically derived solution of fluid flowing through a channel with flexible walls being perturbed by a traveling wave.

\section{Data Availability}

The present paper is concerned with an important question on stability of a fluid flow interacting with flexible channel walls. This research has a purely analytical nature; that is, it is devoted to finding a representation for a solution of the initial boundary value problem generated by Navier-Stokes equations in the case of a specifically chosen geometry of the channel and properties of the boundary. Due to the very nature of this research, we have used many mathematical tools (like techniques from the area of ordinary and partial differential equations, boundary value problems, integral transformations, improper integration, evaluation of contour integrals on the complex plane by using the Residue theorem, etc.). The obtained results are theoretical. In our forthcoming work, we are planning to compare analytical results with the experimental data. However, for the present work, no data was necessary.

\section{Conflicts of Interest}

The authors declare that they have no conflicts of interest.

\section{Acknowledgments}

Partial support by the National Science Foundation (Grant no. DMS-810826) is highly appreciated by the first author.

\section{References}

[1] L. Huang, "Reversal of the Bernoulli effect and channel flutter," Journal of Fluids and Structures, vol. 12, no. 2, pp. 131-151, 1998.

[2] K. M. Case, "Stability of inviscid plane Couette flow," Physics of Fluids, vol. 3, no. 2, pp. 143-148, 1960.

[3] K. M. Case, "Hydrodynamic stability and the initial value problem," Proceedings of Symposia in Applied Mathematics, vol. 13, pp. 25-33, 1962.

[4] B. Shivamoggi, "Stability of inviscid plane Couette flow," Acta Mech, vol. 44, no. 3-4, pp. 327-329, 1982.
[5] P. W. Carpenter and A. D. Garrad, "The hydrodynamic stability of flow over Kramer-type compliant surfaces. Part 2. flow-induced surface instabilities," Journal of Fluid Mechanics, vol. 170, pp. 199-232, 1986.

[6] V. Kumaran, "Stability of inviscid flow in a flexible tube," Journal of Fluid Mechanics, vol. 320, no. -1, pp. 1-17, 1996.

[7] V. Kumaran, "Stability of the flow of a fluid through a flexible tube at intermediate Reynolds number," Journal of Fluid Mechanics, vol. 357, pp. 123-140, 1998.

[8] P. Drazin and W. Reid, Hydrodynamic Stability 124, 1981.

[9] M. Heil and O. E. Jensen, "Flows in deformable tubes and channels," Flow Past Highly Compliant Boundaries and in Collapsible Tubes, vol. 75, pp. 15-49, 2003.

[10] S. I. Abdelsalam and M. M. Bhatti, "Anomalous reactivity of thermo-bioconvective nanofluid towards oxytactic microorganisms," Applied Mathematics and Mechanics, vol. 41, no. 5, pp. 711-724, 2020.

[11] Y. A. Elmaboud and S. I. Abdelsalam, "DC/AC magnetohydrodynamic-micropump of a generalized Burger's fluid in an annulus," Physica Scripta, vol. 94, 2019.

[12] S. I. Abdelsalam and M. Sohail, "Numerical approach of variable thermophysical features of dissipated viscous nanofluid comprising gyrotactic micro-organisms Pramana -," Journal of Physics, 2020.

[13] S. I. Abdelsalam, K. S. Mekheimer, and A. Z. Zaher, "Alterations in blood stream by electroosmotic forces of hybrid nanofluid through diseased artery: aneurysmal/stenosed segment Chinese," Journal of Physics, 2020.

[14] R. M. Abumandour, I. M. Eldesoky, M. H. Kamel, M. M. Ahmed, and S. I. Abdelsalam, "Peristaltic thrusting of a thermal-viscosity nanofluid through a resilient vertical pipe," A Journal of Physical Science, 2020.

[15] M. M. Bhatti, M. Marin, A. Zeeshan, R. Ellahi, and S. I. Abdelsalam, "Swimming of motile gyrotactic microorganisms and nanoparticles in blood flow through anisotropically tapered arteries," Frontier Physics, 2020.

[16] M. M. Bhatti, M. Marin, A. Zeeshan, and S. I. Abdelsalam, "Editorial: recent trends in computational fluid dynamics," Frontier Physics, vol. 8, pp. 1-4, 2020.

[17] I. M. Eldesoky, S. I. Abdelsalam, W. A. El-Askary, and M. M. Ahmed, "The integrated thermal effect in conjunction with slip conditions on peristaltically induced particle-fluid transport in a catheterized pipe," Journal of Porous, 2020.

[18] I. M. Eldesoky, S. I. Abdelsalam, W. A. El-Askary, A. M. ElRefaey, and M. M. Ahmed, "Joint effect of magnetic field and heat transfer on particulate fluid suspension in a catheterized wavy tube,” Bionanoscience, vol. 9, 2019.

[19] H. Sadaf and S. I. Abdelsalam, "Adverse effects of a hybrid nanofluid in a wavy non-uniform annulus with convective boundary conditions," RSC Advances, vol. 10, no. 26, pp. 15035-15043, 2020.

[20] M. Sohail, R. Naz, and S. I. Abdelsalam, “Application of nonFourier double diffusions theories to the boundary-layer flow of a yield stress exhibiting fluid model," Physica A: Statistical Mechanics and Its Applications, vol. 537, p. 122753, 2020.

[21] T. Aittokallio, M. Gyllenberg, and O. Polo, "A model of a snorer's upper airway Turku Cent," Composites Science and Technology, vol. 266, 1999.

[22] R. Beck, M. Odeh, A. Oliven, and N. Gavriely, "The acoustic properties of snores," European Respiratory Journal, vol. 8, no. 12 , pp. $2120-2128,1995$.

[23] J. B. Grotberg and N. Gavriely, "Flutter in collapsible tubes: a theoretical model of wheezes," Journal of Applied Physiology, vol. 66, no. 5, pp. 2262-2273, 1989. 
[24] Y. Aurégan and C. Depollier, "Snoring: linear stability analysis andin-vitroexperiments," Journal of Sound and Vibration, vol. 188, no. 1, pp. 39-53, 1995.

[25] Z. S. Liu, X. Y. Luo, H. P. Lee, and C. Lu, "Snoring source identification and snoring noise prediction," Journal of Biomechanics, vol. 40, no. 4, pp. 861-870, 2007.

[26] D. Pevernagie, R. M. Aarts, and M. De Meyer, "The acoustics of snoring," Sleep Medicine Reviews, vol. 14, no. 2, pp. 131-144, 2010.

[27] J. Wang, G. Tetlow, and A. Lucey, "Flow-structure interaction in the upper airway: motions of a cantilevered flexible plate in channel flow with flexible walls," in Proceedings of the 16th Australasian Fluid Mechanics Conference, pp. 342-345, Gold Coast, Australia, January 2007.

[28] R. J. Whittaker, M. Heil, O. E. Jensen, and S. L. Waters, "Predicting the onset of high-frequency self-excited oscillations in elastic-walled tubes," Proceedings of the Royal Society A: Mathematical, Physical and Engineering Sciences, vol. 466, no. 2124 , pp. $3635-3657,2010$.

[29] K. Karagiozis, M. Païdoussis, M. Amabili, and A. Misra, "Nonlinear stability of cylindrical shells subjected to axial flow: theory and experiments," Journal of Sound and Vibration, vol. 309, no. 3-5, pp. 637-676, 2008.

[30] M. Païdoussis, "Some unresolved issues in fluid-structure interactions," Journal of Fluids and Structures, vol. 20, no. 6, pp. 871-890, 2005.

[31] M. P. Païdoussis and J.-P. Denise, "Flutter of thin cylindrical shells conveying fluid," Journal of Sound and Vibration, vol. 20, no. 1, pp. 9-26, 1972.

[32] M. P. Païdoussis and T. P. Luu, "Dynamics of a pipe aspirating fluid such as might be used in ocean mining," Journal of Energy Resources Technology, vol. 107, no. 2, pp. 250-255, 1985.

[33] P. G. Larose and J. B. Grotberg, "Flutter and long-wave instabilities in compliant channels conveying developing flows," Journal of Fluid Mechanics, vol. 331, pp. 37-58, 1997.

[34] M. Païdoussis and G. Li, "Pipes conveying fluid: a model dynamical problem," Journal of Fluids and Structures, vol. 7, no. 2, pp. 137-204, 1993.

[35] M. P. Païdoussis, "Aspirating pipes do not flutter at infinitesimally small flow," Journal of Fluids and Structures, vol. 13, no. 3, pp. 419-425, 1999.

[36] M. P. Païdoussis, C. Semler, and M. Wadham-Gagnon, "A reappraisal of why aspirating pipes do not flutter at infinitesimal flow," Journal of Fluids and Structures, vol. 20, no. 1, pp. 147-156, 2005.

[37] M. Amabili, F. Pellicano, and M. P. Païdoussis, "Non-linear dynamics and stability of circular cylindrical shells containing flowing fluid. Part I: stability," Journal of Sound and Vibration, vol. 225, no. 4, pp. 655-699, 1999.

[38] L. Huang, "Viscous flutter of a finite elastic membrane in Poiseuille Flow," Journal of Fluids and Structures, vol. 15, no. 7, pp. 1061-1088, 2001.

[39] M. A. Shubov and M. M. Edwards, Mathematical Analysis of a Model of Blood Flow through a Channel with Flexible Walls, University New Hampshire, Hanover, NH, USA, 2020.

[40] G. F. Roach, Green's Functions, Cambridge University Press, Cambridge, MA, USA, 2nd edition, 1995.

[41] I. Stakgold, Green's Functions and Boundary Value Problems, John Wiley \& Sons, Hoboken, NJ, USA, 2011. 